[Article]

www.whxb.pku.edu.cn

\title{
锈层下碳钢的腐蚀电化学行为特征
}

\author{
邹姘 ${ }^{1}$ 王 佳 ${ }^{1,2, *}$ 郑莹莹 ${ }^{1}$ \\ (' 中国海洋大学化学化工学院, 山东青岛 266100; \\ 2 中国科学院金属研究所, 金属腐蚀与防护国家重点实验室, 沈阳 110016)
}

\begin{abstract}
摘要: 采用极化曲线、线性极化电阻(LPR)和电化学阻抗(EIS)研究了海水中带锈碳钢的电化学行为, 结果发现: 长期浸泡的内锈层对电极过程有较大影响; 短期浸泡, LPR 和 EIS 测定的极化电阻 $\left(R_{\mathrm{p}}\right)$ 逐渐增大; 而长期浸泡, $R_{\mathrm{p}}$ 却逐渐减小; 随着浸泡时间的延长, $R_{\mathrm{p}}$ 出现了先增大后减小的变化趋势. 将锈层逐层剥离后研究了碳钢的电化学 行为, 并结合傅里叶变换红外 (FTIR)光谱和横截面结构分析表明, 这主要是因为长期浸泡后, 内锈层中出现了具 有较高电化学活性的 $\beta$ - $\mathrm{FeOOH}$, 并且其含量随着浸泡时间的延长而逐渐增加. 当进行电化学测试时, 在对体系 进行一定程度极化的过程中, $\beta-\mathrm{FeOOH}$ 参与了阴极还原反应, 使电极过程不再是简单的阳极金属溶解和阴极氧 还原, 加快了阴极反应速度, 从而导致 $R_{\mathrm{p}}$ 逐渐减小.
\end{abstract}

关键词：电化学行为; 锈层; 极化电阻; 阴极还原; 傅里叶变换红外光谱

中图分类号: $\mathrm{O} 646$

\section{Electrochemical Corrosion Behaviors of Rusted Carbon Steel}

\author{
ZOU Yan ${ }^{1} \quad$ WANG Jia ${ }^{1,2, *} \quad$ ZHENG Ying-Ying ${ }^{1}$ \\ ('College of Chemistry and Chemical Engineering, Ocean University of China, Qingdao 266100, Shandong Province, \\ P. R. China; ${ }^{2}$ State Key Laboratory for Corrosion and Protection, Institute of Metal Research, \\ Chinese Academy of Sciences, Shenyang 110016, P. R. China)
}

\begin{abstract}
Electrochemical methods including polarization curves, linear polarization resistance (LPR), and electrochemical impedance spectroscopy (EIS) were used to characterize and investigate the electrochemical behavior of rusted carbon steel immersed in seawater. Results indicate that the inner rust layer that forms on the surface of the carbon steel after long-term immersion greatly affects the electrode process. Polarization resistance $\left(R_{\mathrm{p}}\right)$, determined by LPR and EIS, increases during the initial immersion period. After long-term immersion, it decreases. $R_{\mathrm{p}}$ initially increases and then decreases gradually with immersion time. The electrochemical characteristics of the rusted carbon steel were studied by removing the outer and inner rust layers. The materials were analyzed by Fourier transform infrared (FTIR) spectroscopy and their cross-sectional morphologies were obtained to determine the cause. The results show that the $\beta$ - $\mathrm{FeOOH}$, which exists in the inner rust layer, has high electrochemical activity. Its content increases with the growth of the inner rust layer. In the electrochemical tests, even a small amount of polarization allows $\beta$-FeOOH to participate in the cathodic reduction reaction. Besides the anodic dissolution of iron and the cathodic reduction of oxygen, rust reduction is also possible. For this reason, the cathodic reaction rate is promoted and $R_{\mathrm{p}}$ decreases.
\end{abstract}

Key Words : Electrochemical behavior; Rust layer; Polarization resistance; Cathodic reduction; Fourier transform infrared spectroscopy

Received: February 21, 2010; Revised: March 29, 2010; Published on Web: June 25, 2010.

"Corresponding author. Email: jwang@ouc.edu.cn; Tel: +86-532-66781903.

The project was supported by the National Natural Science Foundation of China (50971118).

国家自然科学基金(50971118)资助项目

(C) Editorial office of Acta Physico-Chimica Sinica 
锈层/金属是多相多界面复杂腐蚀体系之一, 其 特征是腐蚀过程涉及多个相内传质和多个相界面区 迁移反应, 并且腐蚀进程也会随着腐蚀产物积累而 增减和转化. 复杂腐蚀体系的上述特征导致测量方 法和腐蚀数据变得复杂和难于解析, 增加了研究工 作的不确定性. 锈层下的腐蚀是复杂的电化学过程, 锈层覆盖金属的腐蚀电化学反应涉及锈层自身氧化 还原, 锈层相内传质, 锈层相界面电荷迁移以及多孔 锈层中微生物繁殖等多个子过程组成的复杂腐蚀 过程 ${ }^{[1-3]}$, 导致电极过程复杂化.

碳钢是海洋环境中使用的最主要的金属结构材 料, 其腐蚀一般为均匀腐蚀, 锈层下碳钢的腐蚀是最 主要的、持续时间最长的腐蚀形态, 生成的铁锈主要 是与铁的腐蚀有关的氧化物和羟基氧化物. 锈层下 的腐蚀行为与许多因素有关, 如锈层的形成机制、锈 层的发展变化和锈层的组成结构等. 在不同环境下 生成的锈层对碳钢的电极过程有不同程度的影响, 因此研究其腐蚀产物与电化学行为的关系极为重 要.

García 等 ${ }^{[4]}$ 认为碳钢全浸在海水中, 在其表面生 成的锈层可以分为两层: 紧密附着的内锈层和附着 不牢固的外锈层. 外锈层中 $\gamma-\mathrm{FeOOH}$ 的含量最多, 而内锈层中尖晶石相 (磁性 $\mathrm{Fe}_{3} \mathrm{O}_{4}$ 和磁性 $\mathrm{Fe}_{2} \mathrm{O}_{3}$ ) 的含 量最多. $\beta$ - $\mathrm{FeOOH}$ 主要在金属的表面形成并紧紧附 着在金属表面, 使锈层具有多孔性并且成分不均匀, 因而不具有保护性. Ma 等 ${ }^{[5}$ 研究了在海洋大气中腐 蚀生成的碳钢锈层, 发现在空气中暴露 3 个月以后 锈层开始分为两层: 外层从 6 个月开始组成不变, 由 $\alpha-\mathrm{FeOOH} 、 \gamma-\mathrm{FeOOH}$ 、无定形的氢氧化物和 $\mathrm{Fe}_{3} \mathrm{O}_{4}$ 组成; 6 个月时内层只有 $\beta-\mathrm{FeOOH}$ 和无定形的氢氧 化物, 腐蚀速度增大; 暴露 24 个月, 内锈层由 $\alpha$ $\mathrm{FeOOH} 、 \gamma-\mathrm{FeOOH}$ 、无定形的氢氧化物和 $\mathrm{Fe}_{2} \mathrm{O}_{3}$ 组成, 腐蚀速度减小. $\alpha-\mathrm{FeOOH}$ 是一种比较稳定的物质, 具有比较低的电化学活性, 在锈层中可以起到比较 好的保护作用 ${ }^{[6}$. Stratmann 等 ${ }^{[7-10]}$ 研究了在干湿循环 条件下纯铁的腐蚀行为, 认为锈层中 $\gamma-\mathrm{FeOOH}$ 与 $\mathrm{Fe}$ 的腐蚀电位比较接近, $\gamma$ - $\mathrm{FeOOH}$ 是电化学活性物 质, 可以作为阴极参与还原反应. Nishimura 等 ${ }^{[11]}$ 认 为在含有 $\mathrm{Cl}^{-}$环境中形成 $\beta-\mathrm{FeOOH}$ 很容易发生电化 学还原反应.

近年来发现, 在金属/锈层复杂体系中, 由于其 复杂的电极过程而导致电化学方法测定的极化电阻 $\left(R_{\mathrm{p}}\right)$ 也受到了锈层的影响. Andrade 等 ${ }^{[12]}$ 在用电化学
阻抗法研究钢筋在混凝土中的电化学行为时发现, 在电位 $-0.7 \mathrm{~V}(v s \mathrm{SCE})$ 附近, $R_{\mathrm{p}}$ 会受腐蚀产物氧化还 原反应的影响. González 等 ${ }^{[13]}$ 发现用传统的极化电 阻方法不适合测定带锈金属的腐蚀电流密度. 当锈 层存在时, 锈层中二价和三价铁离子的氧化还原反 应会产生很大的偏离正常双电层电容数值的假电 容. 锈层一方面能给阴极反应提供还原物质, 另一方 面可以作为氧气还原的多孔电极. Flis 等 ${ }^{[14]}$ 认为钢 筋经过长时间腐蚀后, 界面转输电阻 $R_{\mathrm{ct}}$ 减小, 这主 要是因为高价态的腐蚀产物被还原. Videm ${ }^{[15]}$ 用循 环伏安、恒电位阶跃、恒电流阶跃、电化学阻抗等电 化学方法测量了钢在碱性溶液中的腐蚀速度, 发现 各种电化学方法测得的结果之间有很大分歧, 主要 原因是腐蚀产物的氧化还原反应掩盖了金属发生腐 蚀反应的法拉第电流.

为此, 本文采用多种电化学方法研究了海水中 锈层下碳钢的电化学行为和在自腐蚀电位附近的电 极过程特征, 结合锈层的组成结构分析, 探讨了在电 化学测试过程中碳钢阴极和阳极的反应机理.

\section{1 实验方法 \\ 1.1 电极材料}

实验材料采用 Q235 钢, 其化学成分 (质量分数 $(w), \%)$ 为: C 0.18, Si 0.30, Mn 0.32, P 0.04, S 0.04, Fe 余量. 电化学试样为厚度 $3 \mathrm{~mm}$ 、直径 $10 \mathrm{~mm}$ 的圆 柱, 一端为工作面, 其余面用环氧树脂(莱阳经济技 术开发区精细化工厂)封装. 在实验前将所有试样用 $400 \# 、 600 \#$ 和 800 水磨砂纸逐级打磨, 然后用去离 子水清洗, 酒精除油后在干燥器中备用. 实验开始 时, 将电化学样品浸泡在室内海水试验箱(深圳又昭 实业有限公司)中. 实验所用海水取自青岛汇泉湾, 室内的平均温度为 $20{ }^{\circ} \mathrm{C}$, 在实验箱中的海水为静态 海水, 每个星期更换海水一次. 实验开始于 2007 年 11 月, 定期进行电化学测试.

\section{2 红外光谱测试}

用毛刷刷下外层疏松的腐蚀产物, 再用刀片小 心剥离内锈层, 将取下的外层和内层腐蚀产物研成 粉末, 放人真空干燥器中以备红外测试. 分析仪器为 美国 Nicolet 公司生产的 Nexus-380 型傅里叶变换 红外光谱仪, 测量光谱区域为 $400-1300 \mathrm{~cm}^{-1}$, 设定 扫描次数为 32 次, 实际分辨率为 $4 \mathrm{~cm}^{-1}$. 取少量锈 层样品进行测量, 加人适量的 $\mathrm{KBr}$ 粉末, 充分研细 并混合均匀, 利用模具加压制成透明样品后置于光 
谱仪样品室进行测试. 横截面形貌采用深圳 Jetet 公 司生产的 JT-2182II 彩色摄像机进行拍摄.

\section{3 电化学测试}

电化学测试采用三电极体系, 辅助电极为铂片 电极, 参比电极为饱和甘录电极, 电解液为海水, 使 用美国 Princeton 公司生产的 PARSTAT 2263 电化 学工作站进行腐蚀电化学测量, 测试软件为 Powersuite(美国 Princeton). 分别剥下不同锈层进行电化 学测试的剥离方法与进行红外测试锈层的剥离方法 相同. 电化学阻抗(EIS)测试是在自腐蚀电位下进行 的, 频率范围为 $9 \mathrm{kHz}-9 \mathrm{mHz}$, 交流激励信号幅值为 $10 \mathrm{mV}$. 线性极化电阻 (LPR) 测试的扫描速率为 0.1 $\mathrm{mV} \cdot \mathrm{s}^{-1}$. 扫描范围为相对于自腐蚀电位 $\pm 10 \mathrm{mV}$. 极 化曲线测试扫描速率为 $0.1 \mathrm{mV} \cdot \mathrm{s}^{-1}$, 为了避免锈层 的影响, 极化曲线分别从自腐蚀电位向阴极和阳极 扫描. 极化曲线每次采用 3 个平行试样, 挑选具有代 表性的实验结果; 电化学阻抗测试和线性极化测试 采用 5 个平行试样, 每次都对这 5 个试样进行测试, 选取具有代表性的一组实验结果. 测试时溶液的平 均温度为 $20^{\circ} \mathrm{C}$.
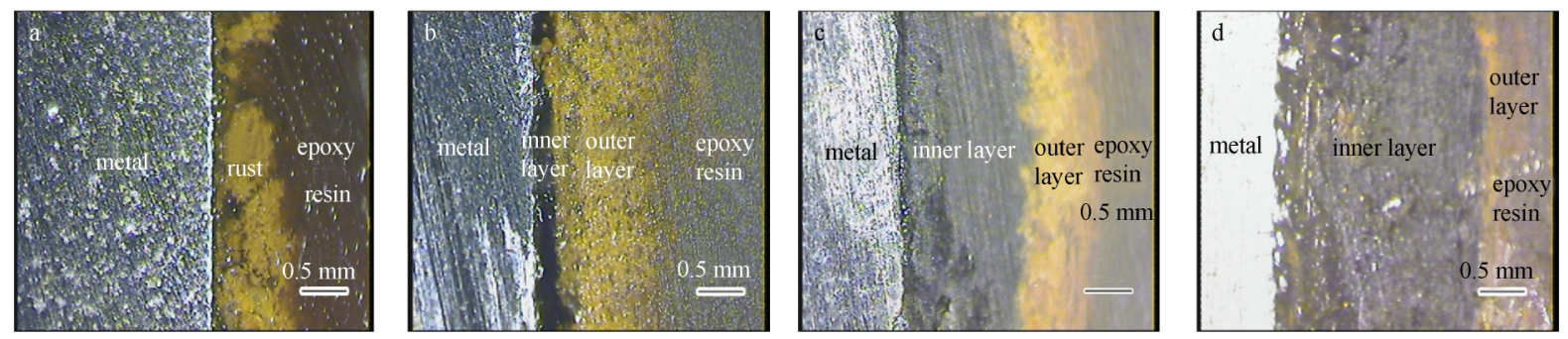

图 1 在海水中浸泡不同时间 Q235 钢的横截面形貌

Fig.1 Morphologies of cross-section of Q235 steel immerged in seawater for various time

(a) 6 weeks, (b) 11 weeks, (c) 18 weeks, (d) 48 weeks
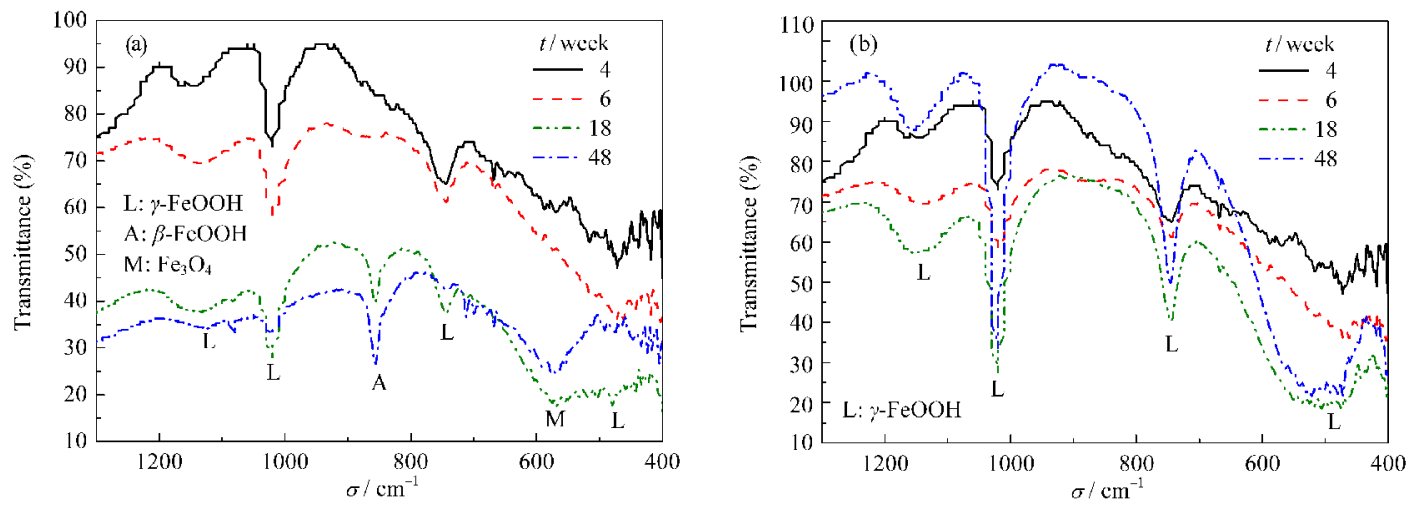

图 2 浸泡不同时间 Q235 钢锈层的 FTIR 光谱图

Fig.2 FTIR spectra of rust layers for Q235 steel for various immersion time (a) inner layer; (b) outer layer 


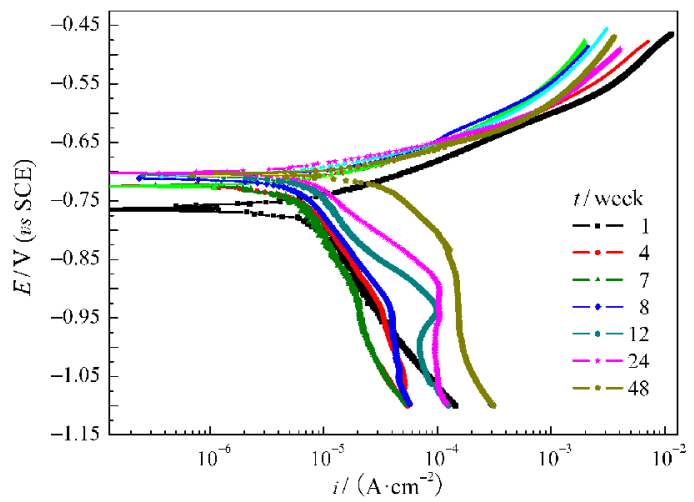

图 3 在海水中浸泡不同时间 Q235 钢的极化曲线

Fig.3 Polarization curves of Q235 steel immerged in seawater for various time

$\gamma-\mathrm{FeOOH}$, 但没有 $\beta-\mathrm{FeOOH}$. 内锈层除了 $\gamma-\mathrm{FeOOH}$ 的特征峰外, $\beta-\mathrm{FeOOH}$ 的特征峰变得很强, 说明 $\beta$ $\mathrm{FeOOH}$ 的含量明显增多. 另外, 在 $587 \mathrm{~cm}^{-1}$ 还有一 个比较宽的特征峰, 这与 $\mathrm{Fe}_{3} \mathrm{O}_{4}$ 的特征峰相吻合. 浸 泡 48 周后, 外锈层的成分没有发生变化, 内锈层中 $\beta-\mathrm{FeOOH}$ 的特征峰强度超过了 $\gamma-\mathrm{FeOOH}$. 这些结果 表明, 浸泡前期没有分层时的锈层组分与浸泡后期 分层后的外锈层组分相同; 8 周前基本上不存在 $\beta$ $\mathrm{FeOOH}$, 它主要形成于 8 周之后的内锈层中, 其含 量随着浸泡时间的增加而逐渐增大.

\section{3 锈层下碳钢的电化学行为}

\subsection{1 极化曲线行为}

图 3 为不同浸泡时间碳钢的稳态极化曲线. 随 着浸泡时间的增加, 自腐蚀电位逐渐正移, 4 周以后 自腐蚀电位基本稳定. 阳极反应速度和斜率基本不 变表明锈层对阳极过程的影响不大, 锈层下阳极过 程仍然是铁溶解反应. 但是, 锈层对阴极过程有较大 影响. 相比之下, 8 周前阴极行为变化很小, 从 8 周 开始阴极极限电流平台和阴极反应速度逐渐增加, 阴极的反应机理也应该随之发生变化.

\subsection{2 线性极化行为}

图 4 为海水中浸泡 48 周碳钢电极的线性极化 曲线. 浸泡初期自腐蚀电位较负, 随着浸泡时间的增 加, 自腐蚀电位正移并逐渐稳定, 线性极化曲线也略 微有点弯曲, 这是由于腐蚀产物在碳钢表面的累积 造成的. 因此, 拟合区域选在自腐蚀电位附近接近直 线段的 $\pm 5 \mathrm{mV}$ 范围内. 测定的 $R_{\mathrm{p}}$ 变化见图 5 .

$R_{\mathrm{p}}$ 随时间变化的主要特征是 8 周以前 $R_{\mathrm{p}}$ 持续 增加, 从第 8 周开始突然转为迅速减小, 12 周以后 逐渐趋于平缓. 8 周前后存在截然不同的行为这一

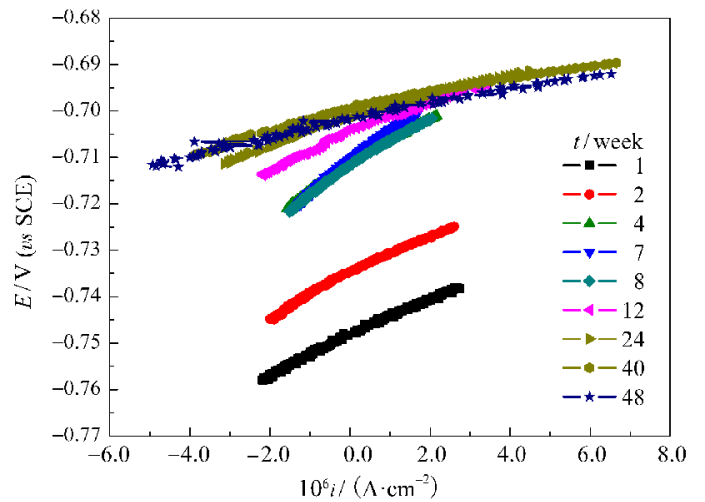

图 4 浸泡不同时间 Q235 钢的线性极化曲线

Fig.4 Linear polarization curves of $\mathbf{Q 2 3 5}$ steel immerged in seawater for various time

特征与图 3 的极化曲线变化是一致的, 表明这也是 阴极过程的增强引起的. 由此可以表明, 长期浸泡的 锈层在电化学测试过程中对自腐蚀电位附近的电极 过程也产生了较大的影响.

\subsection{3 电化学阻抗行为}

图 6 为浸泡 48 周碳钢电极的电化学阻抗响应. Nyquist 图(图 6(a、b)) 是由两个下偏的半圆构成, 可 能是电极表面的粗粘度导致了充放电过程的不均 匀 ${ }^{[16]}$, 因而用常相位角元件 $Q$ 来代替电容元件 $C$. 高频反映了锈层的性质, 低频反映了锈层/金属界面 的电极过程. 随着锈层的增厚, 最大幅角对应的频率 向低频方向移动, Bousselmi 等 ${ }^{[2]}$ 认为是锈层厚度的 增加导致了这种变化. 碳钢在海水中浸泡一段时间 后生成的锈层可以分为内锈层和外锈层, 而从极化 曲线和 LPR 测试中发现 8 周前后电化学行为发生 较大变化, 这也可能与内锈层的生成有关, 因此浸泡 前 8 周用等效电路图 7(a) ${ }^{[2]}$ 来拟合, 8 周以后(包括 8 周)等效电路见图 7(b) ${ }^{[5,17]}$, 证实拟合的曲线与实验曲

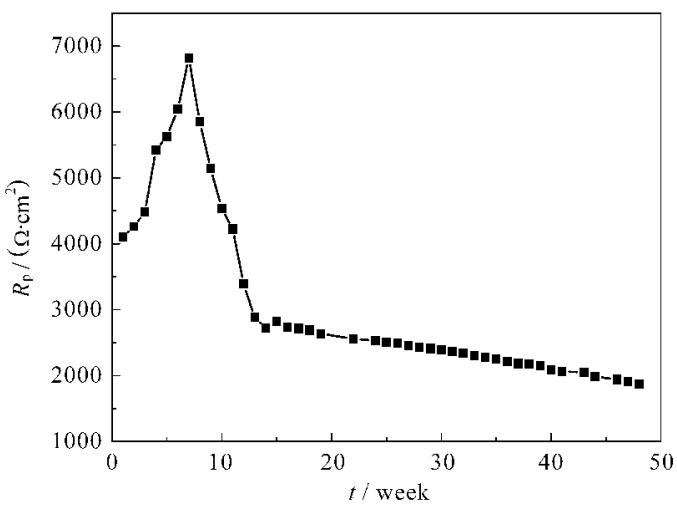

图 5 线性极化法测定的极化电阻 $\left(\boldsymbol{R}_{\mathrm{p}}\right)$ 随时间的变化 Fig.5 Polarization resistance $\left(R_{\mathrm{p}}\right)$ measured by LPR with increasing of immersion time 

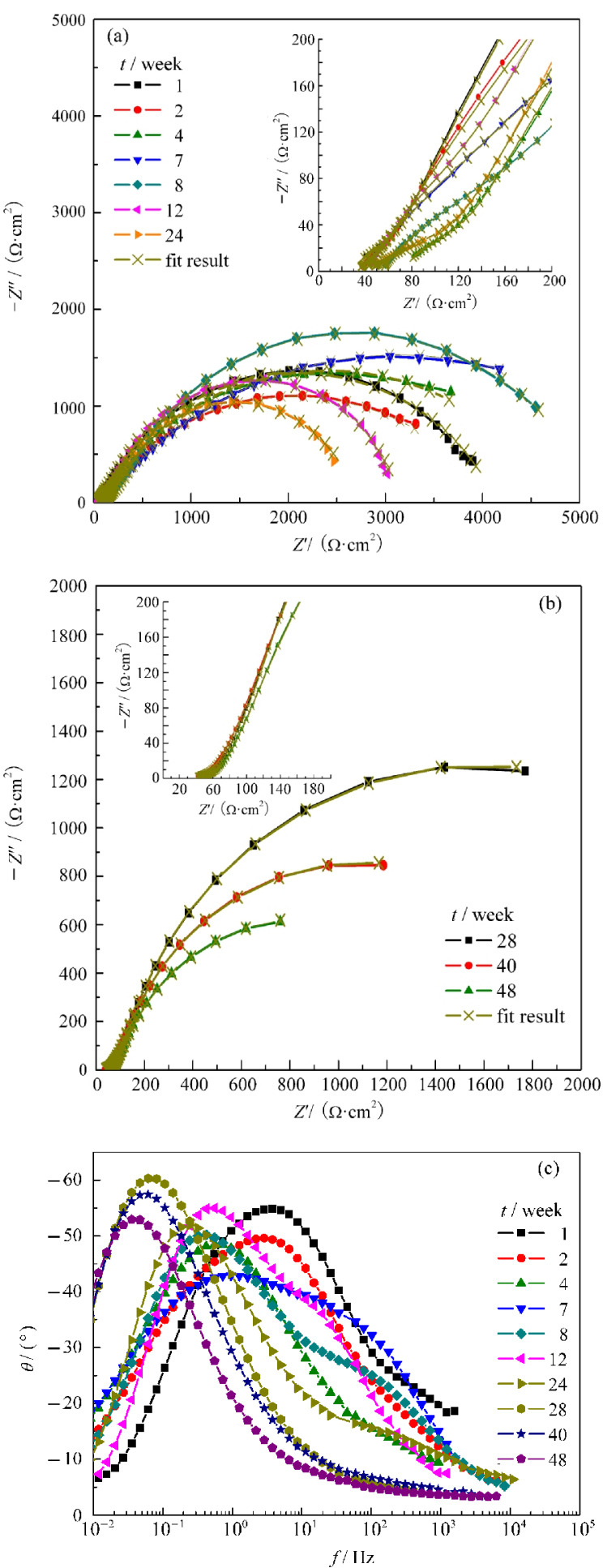

图 6 在海水中浸泡不同时间 Q235 钢的阻抗图

Fig.6 EIS diagrams of Q235 steel immerged in seawater for various time

(a, b) Nyquist diagram, (c) Bode diagram; The inset figure is the enlarged diagram of high frequency.

线吻合良好, 误差在 $5 \%$ 以内.

由于阴极反应是腐蚀过程的控制步骤, 因此极 化电阻 $R_{\mathrm{p}}$ 反映了在金属/锈层界面发生阴极反应的
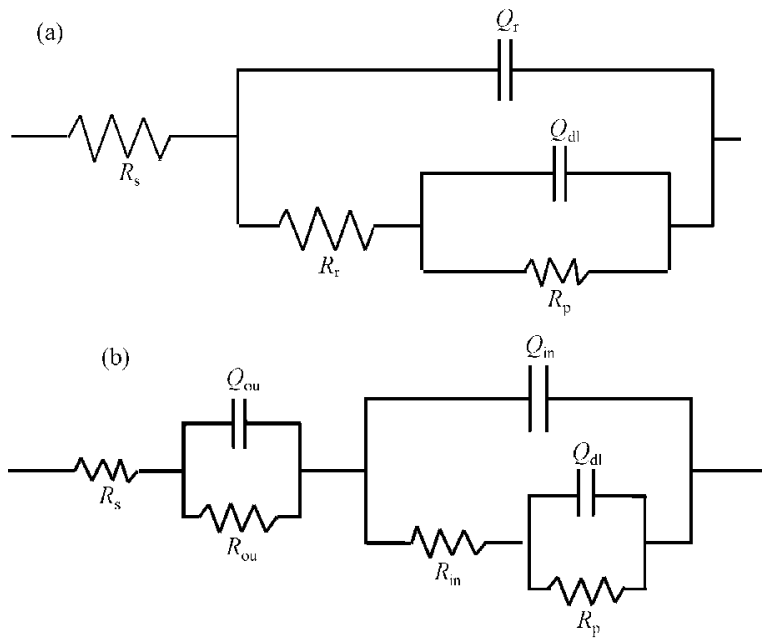

图 7 在海水中浸泡不同时间 Q235 钢的等效电路

Fig.7 Equivalent circuits of Q235 steel immerged in seawater for various time

(a) before immerging for 8 weeks ${ }^{[2]}$, (b) after immerging for 8 weeks ${ }^{[5,17]}$ (including 8 weeks); $R_{\mathrm{s}}$ : resistance of solution; $R_{\mathrm{r}}$ and $Q_{\mathrm{r}}$ : resistance and capacitance of the rust layer; $R_{\mathrm{ou}}$ and $Q_{\mathrm{ou}}$ : resistance and capacitance of the outer rust layer; $R_{\mathrm{in}}$ and $Q_{\mathrm{in}}$ : resistance and capacitance of the inner rust layer; $R_{\mathrm{p}}$ : polarization resistance; $Q_{\mathrm{d}}$ : capacitance of the electric double layer
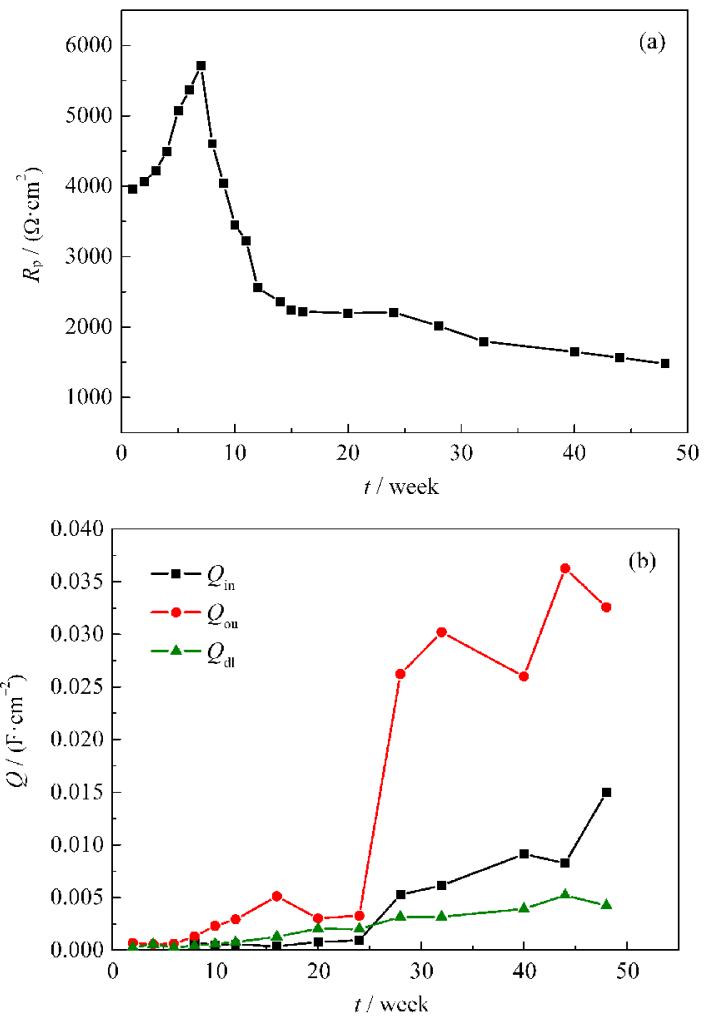

图 8 EIS 测定的极化电阻和电容随浸泡时间的变化

Fig.8 $R_{\mathrm{p}}(\mathrm{a})$ and capacitances (b) measured by EIS with increasing of immersion time

能力. 随着浸泡时间的增加, $R_{\mathrm{p}}$ 逐渐增大; 8 周以后, $R_{\mathrm{p}}$ 转为逐渐减小, 12 周以后变化缓慢(图 8(a)). EIS 

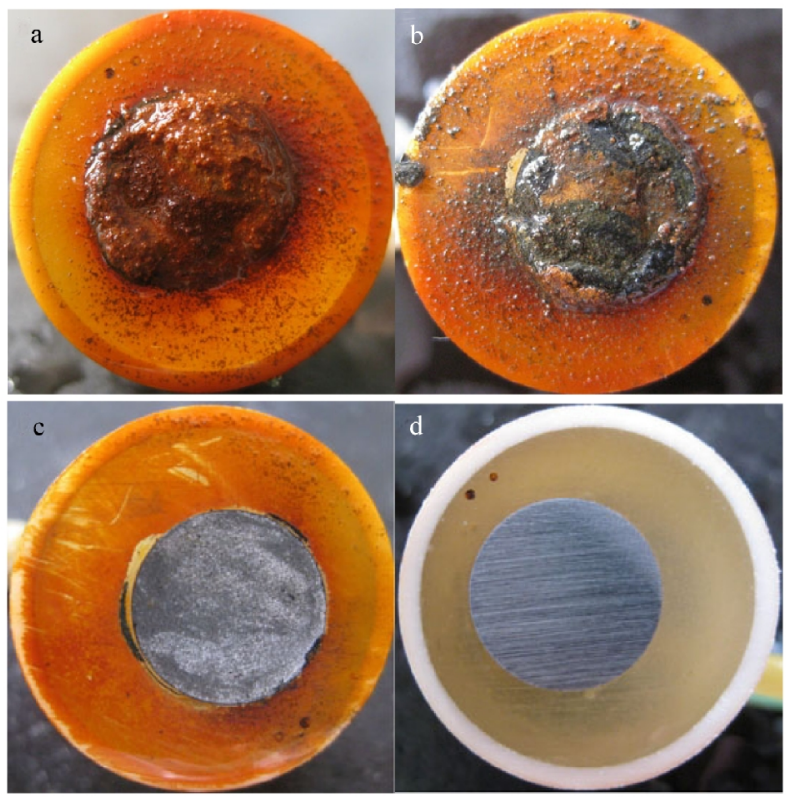

图 9 在海水中浸泡 20 周 Q235 钢不同锈层覆盖的 电极形貌

Fig.9 Appearances of Q235 steel with different rust layers after immerging in seawater for 20 weeks

(a) rust covered; (b) removal of the outer layer;

(c) removal of the inner layer; (d) naked coupons

方法得到的 $R_{\mathrm{p}}$ 变化趋势与 $\mathrm{LPR}$ 方法是一致的. 从 电容的数值来看(图 8(b)), 浸泡前 24 周, 内、外锈层 电容和双电层电容变化都不大, 而这段时间 $R_{\mathrm{p}}$ 却经 历了先增大后减小的过程; 24 周以后内锈层和外锈 层的锈层电容都显著增加, 而极化电阻却变化不大, 这说明了比较大的锈层电容虽然在一定程度上会影 响 $R_{\mathrm{p}}$ 的测定, 但不一定是主要因素, 有可能是反应 机理发生变化而导致了长期浸泡的 $R_{\mathrm{p}}$ 逐渐减小.

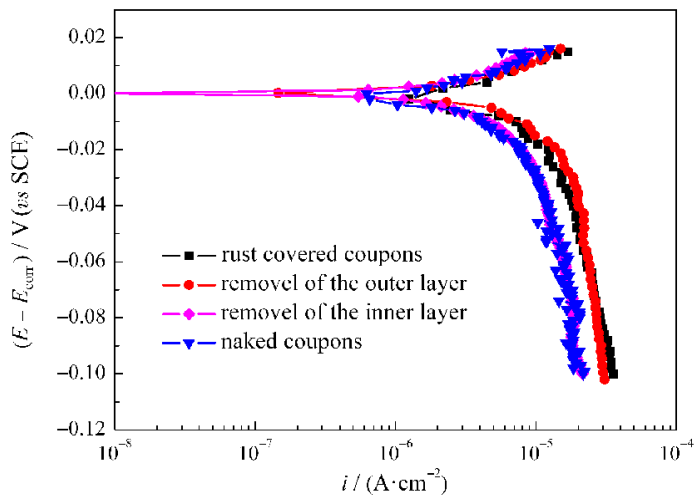

图 10 在海水中浸泡 20 周分别除去外锈层和内锈层的 阴极极化曲线放大图

Fig.10 Magnified cathodic polarization curves of Q235 steel whose outer and inner rust layers were removed separately after immerging in seawater for 20 weeks

\section{4 不同深度锈层的电化学响应特征}

电极表面状态分为以下 4 种状态: 带锈、去除 外锈层、去除内层锈和打磨光滑的裸碳钢 (图 9). 带 锈的碳钢从表面看呈黄色(图 9a 中较浅的颜色), 剥 离外锈层后碳钢表面为黑色(图 $9 \mathrm{~b}$ 中较深的颜色), 剥离内锈层后碳钢表面能看到腐蚀后的蚀坑. 显然, 除掉内锈层的碳钢和光滑的碳钢的阴极行为是相同 的, 仅仅清除外锈层的碳钢和带锈碳钢的阴极行为 是相同的, 同时比除掉内层锈碳钢和光洁碳钢阴极 电流密度显著增大. 这一结果表明, 内锈层是导致阴 极反应加速的主要原因. 图 10 为将在海水中浸泡 20 周带锈电极逐层剥离锈层后的局部阴极极化曲 线.

图 11 为在海水中浸泡 20 周的带锈碳钢逐层剥 离锈层后的电化学阻抗图. 除掉内锈层的碳钢和打 磨光滑的裸碳钢试样具有相同的较高阻抗值, 而被 完整锈层覆盖的碳钢电极和除去外锈层的碳钢电极 具有较低的相同的阻抗值.

这一行为与图 10 的结果是一致的. 碳钢电极表 面被完整锈层覆盖时的电化学阻抗谱的等效电路见 图 7(b). 除去外锈层后只覆盖着内锈层的等效电路 见图 7(a). 除去内锈层和打磨光滑的裸碳钢只有一 个时间常数, 在金属/溶液界面只发生铁的阳极溶解 和氧气的还原反应, 等效电路为 $R_{\mathrm{s}}\left(Q_{\mathrm{d} \mid} R_{\mathrm{p}}\right)$. 按上述等 效电路分别进行拟合后得到的各种电阻值列于表 1 ,

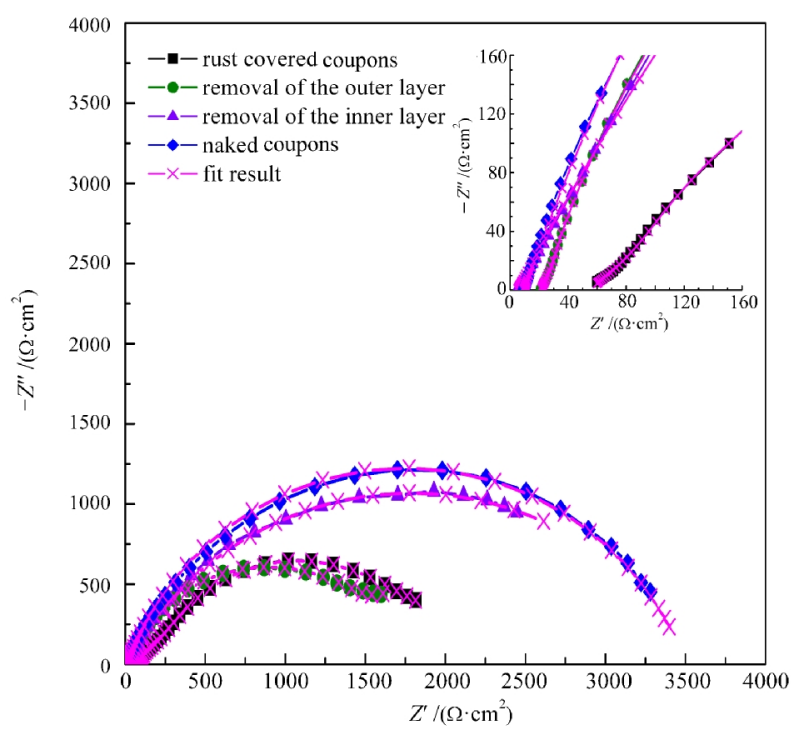

图 11 在海水中浸泡 20 周不同锈层覆盖下的 Q235 钢的 电化学阻抗谱

Fig.11 EIS diagram of Q235 steel with different rust layers after immerging in seawater for 20 weeks The inset figure is the enlarged diagram of high frequency. 
表 1 在海水中浸泡 20 周的不同锈层覆盖下的 Q235 钢的 拟合电阻值

Table 1 Fitted resistance values of Q235 steel with different rust layers after immerging in seawater for 20 weeks

\begin{tabular}{lccc}
\hline & $R_{\mathrm{ou}} /\left(\Omega \cdot \mathrm{cm}^{2}\right) R_{\mathrm{in}} /\left(\Omega \cdot \mathrm{cm}^{2}\right)$ & $R_{\mathrm{p}} /\left(\Omega \cdot \mathrm{cm}^{2}\right)$ \\
\hline rust covered & 195 & 112 & 1466 \\
removal of the outer layer & 0 & 110 & 1473 \\
removal of the inner layer & 0 & 0 & 3281 \\
naked coupons & 0 & 0 & 3258 \\
\hline
\end{tabular}

拟合曲线和实验数据能够很好地吻合(图 11).

去掉外锈层后, 与完整锈层覆盖状态下的 $R_{\mathrm{p}}$ 相 比基本上没有发生变化, 说明外锈层不参与电化学 反应. 除去内锈层后, $R_{\mathrm{p}}$ 反而增大表明在带锈体系 中主要是内锈层加速了阴极反应.

\section{5 电化学测试过程中碳钢的反应机理}

实验中发现, 浸泡前 8 周, 碳钢表面的锈层呈棕 黄色, 没有分层; 8 周以后, 锈层开始分层, 表层为棕 黄色, 里层为黑色.

从锈层组分分析来看, 浸泡 8 周以后锈层的组 成和结构发生了较大的变化. 逐渐形成的内锈层中 由于含有 $\beta-\mathrm{FeOOH}$ 而改变了金属/锈层界面的电化 学过程. Antony 等 ${ }^{[18]}$ 认为 $\gamma-\mathrm{FeOOH}$ 和 $\beta-\mathrm{FeOOH}$ 都 可以发生还原反应, 并且 $\beta$ - $\mathrm{FeOOH}$ 比 $\gamma$ - $\mathrm{FeOOH}$ 更 容易被还原. 当这两种成分共同存在时, $\beta-\mathrm{FeOOH}$ 起主要作用加速腐蚀速度. Lair 等 ${ }^{[19]}$ 将纯的 $\gamma$ $\mathrm{FeOOH}$ 和 $\beta-\mathrm{FeOOH}$ 分别与石墨混合, 制成电极, 外 加阴极电流, 测定了 $\gamma-\mathrm{FeOOH}$ 和 $\beta-\mathrm{FeOOH}$ 在不同 溶液中的还原电位. 结果发现, 在 $0.1 \mathrm{~mol} \cdot \mathrm{L}^{-1} 、 \mathrm{pH}$ 为 7.5 的 $\mathrm{NaCl}$ 溶液中, $\beta-\mathrm{FeOOH}$ 的还原电位为 -400 $\mathrm{mV}$ (vs SHE), 还原效率较高; $\gamma-\mathrm{FeOOH}$ 的还原电位 为 $-450 \mathrm{mV}(\mathrm{vs} \mathrm{SHE})$, 还原效率较低. 在 $0.1 \mathrm{~mol} \cdot \mathrm{L}^{-1}$ 、 $\mathrm{pH}$ 为 8.5 的 $\mathrm{NaCl}$ 溶液中, $\beta-\mathrm{FeOOH}$ 的还原电位为 $-440 \mathrm{mV}$ (vs SHE), $\gamma$ - $\mathrm{FeOOH}$ 的还原电位为 $-510 \mathrm{mV}$ (vs SHE). $\gamma$ - $\mathrm{FeOOH}$ 比 $\beta$ - $\mathrm{FeOOH}$ 的还原电位大约负 50-70 mV, 还原电位越正, 越容易被还原. 从热力学 角度看, $\gamma-\mathrm{FeOOH}$ 具有较低的反应活性, $\beta-\mathrm{FeOOH}$ 具有较高的反应活性.

当进行电化学测试时, 需要对体系进行一定程 度的阴极和阳极极化, 使腐蚀电化学反应偏离腐蚀 电位, 这会导致锈层的电化学反应与非极化状态时 不同, 甚至诱发一些在平衡态时所没有的反应, 致使 进行测定的 $R_{\mathrm{p}}$ 先增大后减小. 因此 8 周前后的锈层
的电化学活性很可能是不同的.

通过极化曲线可以得知, 在浸泡初期, 在金属/ 锈层界面溶解氧的含量较高, 在溶解氧供应比较充 足的情况下, 阴极以氧还原为主, 锈层不会参与阴极 反应 ${ }^{[19]}$. 随着浸泡时间的增加, 内锈层逐渐生成, 锈 层组分开始发生变化. 具有高电化学活性的 $\beta$ $\mathrm{FeOOH}$ 大量出现并且含量逐渐增多. 锈层增厚, 阻 碍作用逐渐增大, 溶解氧越来越难扩散到金属基体 表面, 在电化学测试过程中锈层可以参与还原反应 的能力却逐渐增强, 在金属/锈层界面氧和锈层参与 还原反应的比例不断发生变化. 浸泡初期以氧还原 为主; 一段时间以后 $\beta-\mathrm{FeOOH}$ 开始出现并且含量逐 渐增多, 锈层参与还原反应的比例开始增大, 氧和锈 层共同控制了阴极反应速度; 后期氧含量较少, 而 锈层中却含有大量的 $\beta-\mathrm{FeOOH}$, 阴极以锈层还原为 主, 从而导致了电化学方法测定的 $R_{\mathrm{p}}$ 出现先增大后 减小的情况, 加快了阴极反应速度, 这从极化曲线上 也可以清楚的看到锈层对阴极过程的影响.

根据以上的分析并结合锈层在不同浸泡阶段的 组分, 可以推测出碳钢在电化学测试过程中极化电 阻出现先增大后减小的变化趋势主要和内锈层中 $\beta-\mathrm{FeOOH}$ 的出现及其含量的变化有关. 浸泡前 8 周, 锈层组分中主要是 $\gamma-\mathrm{FeOOH}$, 进行电化学测试时发 生的电化学反应为

阳极: $\mathrm{Fe} \rightarrow \mathrm{Fe}^{2+}+2 \mathrm{e}^{-}$

$$
\mathrm{Fe}^{2+} \rightarrow \mathrm{Fe}^{3+}+\mathrm{e}^{-}
$$

阴极: $\mathrm{O}_{2}+2 \mathrm{H}_{2} \mathrm{O}+4 \mathrm{e}^{-} \rightarrow 4 \mathrm{OH}^{-}$

总的反应方程式为: $4 \mathrm{Fe}+3 \mathrm{O}_{2}+2 \mathrm{H}_{2} \mathrm{O}=4 \mathrm{FeOOH}$

8 周以后, 锈层组分为 $\gamma-\mathrm{FeOOH} \beta-\mathrm{FeOOH}$ 和 $\mathrm{Fe}_{3} \mathrm{O}_{4}$, 进行电化学测试时发生的电化学反应为

阳极: $\mathrm{Fe} \rightarrow \mathrm{Fe}^{2+}+2 \mathrm{e}^{-}$

$$
\mathrm{Fe}^{2+} \rightarrow \mathrm{Fe}^{3+}+\mathrm{e}^{-}
$$

阴极: $\mathrm{O}_{2}+2 \mathrm{H}_{2} \mathrm{O}+4 \mathrm{e}^{-} \rightarrow 4 \mathrm{OH}^{-}$

$$
\mathrm{Fe}^{2+}+8 \mathrm{FeOOH}+2 \mathrm{e}^{-} \rightarrow 3 \mathrm{Fe}_{3} \mathrm{O}_{4}+4 \mathrm{H}_{2} \mathrm{O}
$$

总的反应方程式为:

$$
11 \mathrm{Fe}+8 \mathrm{O}_{2}+4 \mathrm{H}_{2} \mathrm{O}=8 \mathrm{FeOOH}+\mathrm{Fe}_{3} \mathrm{O}_{4}
$$

对于电化学方法来说, 浸泡前 8 周, 在进行电化 学测试时阳极和阴极分别发生铁的溶解和氧的还原 反应, 随着腐蚀产物的增多, 对氧扩散的阻碍能力增 大, 因此测定的 $R_{\mathrm{p}}$ 逐渐增大; 8 周以后, 阴极反应增 加了 $\beta-\mathrm{FeOOH}$ 的还原反应, 增大了阴极电流密度, 从而导致阴极反应加快, $R_{\mathrm{p}}$ 减小. 


\section{3 结 论}

(1) 在海水中碳钢浸泡前 8 周, 锈层没有分层, 主要成分为 $\gamma-\mathrm{FeOOH} ; 8$ 周以后, 内锈层逐渐生成, 主要成分为 $\gamma-\mathrm{FeOOH} \beta-\mathrm{FeOOH}$ 和 $\mathrm{Fe}_{3} \mathrm{O}_{4}$.

(2) 锈层在不同浸泡阶段的组成与结构不同, 导 致锈层下碳钢的阴极行为发生较大变化, 并且使在 自腐蚀电位附近测定的 $R_{\mathrm{p}}$ 先增大后减小.

(3) 长期浸泡生成的内锈层中的 $\beta-\mathrm{FeOOH}$ 具有 较高阴极反应活性, 当进行电化学测试时, 一定程度 的扰动可以使锈层参与阴极还原反应, 加速了阴极 反应过程, 导致了 $R_{\mathrm{p}}$ 逐渐减小.

\section{References}

1 Hou, B. R. Oceanologia et Limnologia Sinica, 1995, 26(5): 514 [侯保荣. 海洋与湖沼, 1995, 26(5): 514]

2 Bousselmi, L.; Fiaud, C.; Tribollets, B.; Triki, E. Corrosion Sci., 1997, 39(9): 1711

3 Duan, J. Z.; Wu, S. R.; Zhang, X. J.; Huang, G. Q.; Du, M.; Hou, B. R. Electrochim. Acta, 2008, 54(1): 22

4 García, K. E.; Morales, A. L.; Barrero, C. A.; Greneche, J. M. Corrosion Sci., 2006, 48(9): 2813

5 Ma, Y. T.; Li, Y.; Wang, F. H. Mater. Chem. Phys., 2008, 112(3): 844
6 Yamashita, M.; Miyuki, H.; Matsuda, Y.; Nagano, H.; Misawa, T. Corrosion Sci., 1994, 36(2): 283

7 Stratmann, M.; Müller, J. Corrosion Sci., 1994, 36(2): 327

8 Stratmann, M.; Bohnenkamp, K.; Engell, H. J. Corrosion Sci., 1983, 23(9): 969

9 Stratmann, M.; HoVmann, K. Corrosion Sci., 1989, 29(11-12): 1329

10 Stratmann, M.; Streckel, H. Corrosion Sci., 1990, 30(6-7): 697

11 Nishimura, T.; Tanaka, I.; Shimizu, Y. Tetsu-to-Hagane, 1995, 81: 1079

12 Andrade, C.; Keddam, M.; Nóvoa, X. R.; Pérez, M. C.; Rangel, C. M.; Takenouti, H. Electrochim. Acta, 2001, 46(24-25): 3905

13 González, J. A.; Miranda, J. M.; Otero, E.; Feliu, S. Corrosion Sci., 2007, 49(2): 436

14 Flis, J.; Pickering, H.W.; Osseo-Asare, K. Electrochim. Acta, 1998, 43(12-13): 1921

15 Videm, K. Electrochim. Acta, 2001, 46(24-25): 3895

16 Panda, B.; Balasubramaniam, R.; Dwivedi, G. Corrosion Sci., 2008, 50(6): 1684

17 Bousselmi, L.; Fiaud, C.; Tribollet, B.; Triki, E. Electrochim. Acta, 1999, 44(24): 4357

18 Antony, H.; Perrin, S.; Dillmann, P.; Legrand, L.; Chaussé, A. Electrochim. Acta, 2007, 52(27): 7754

19 Lair, V.; Antony, H.; Legrand, L.; Chaussé, A. Corrosion Sci., 2006, 48(8): 2050 